\title{
La familia, agente de atención temprana de la niñez prematura: un acompañamiento desde la extensión social
}

\section{Family, an agent of early childhood care in preterm children: social outreach flanking.}

\author{
A família, agente de atenção à primeira infância: um \\ acompanhamento da extensão social.
}

\author{
Ana Lupita Garrido Sandino \\ Universidad Estatal a Distancia \\ San José, Costa Rica \\ agarrido@uned.ac.cr \\ (D) https://orcid.org/0000-0001-7422-7660 \\ Yisley Mariana Morales Piedra \\ Universidad Estatal a Distancia \\ San José, Costa Rica \\ ymoralesp@uned.ac.cr \\ (iD) https://orcid.org/0000-0002-4268-5584 \\ Linda María Madriz Bermúdez \\ Universidad Estatal a Distancia \\ San José, Costa Rica \\ Imadriz@uned.ac.cr \\ (D) https://orcid.org/0000-003-2079-3987
}

Recibido - Received - Recebido: 21/02/2020ＣＣＣｒregido - Revised - Revisado: 18/08/2020Ａceptado - Accepted - Aprovado: 26/08/2020

DOl: https://doi.org/10.22458/ie.v22i33.2823

URL: https://revistas.uned.ac.cr/index.php/innovaciones/article/view/2823

\begin{abstract}
Resumen: La Cátedra de Conceptualización de la Educación Especial de la Universidad Estatal a Distancia desarrolla desde el 2016 el proyecto de extensión "UNED en acción: Promoviendo nuevas oportunidades para la infancia expuesta a factores de riesgo biológico o social". El objetivo es promover acciones educativas para capacitar y empoderar en atención temprana (AT) a las familias o cuidadores de la niñez con factores de riesgo biológico y social entre 0 y 6 años para su desarrollo integral. Se ha orientado a familias de infantes prematuros, considerando que en Costa Rica 10\% de los nacimientos son prematuros (González, 2018). La teoría señala que la AT puede revertir los efectos de este factor de riesgo. Las familias necesitan comprender qué es la AT, pero no siempre pueden acceder a prácticas recomendadas o desconocen su importancia, acentuándose fuera de la Gran Área Metropolitana donde estos servicios son limitados o no existen. La extensión universitaria permite la formación conjunta con las familias, empoderándolas como agentes de cambio del paradigma tradicional de la estimulación
\end{abstract}

\section{(C) (1) $\Theta$}


enmarcado en un modelo clínico donde el especialista tenía el conocimiento, a uno de AT que rompe paradigmas tradicionales. El proyecto asume una línea metodológica de investigación-acción mediante un taller basado en el juego y el aprendizaje en familia. Del 2017 al 2019 se trabajó con 88 participantes en diferentes provincias. El elemento de éxito que favoreció el empoderamiento familiar fue enmarcar el proyecto desde la AT, promoviendo actividades cotidianas como experiencias de aprendizaje, filosofía de la investigación que originó el proceso.

Palabras clave: desarrollo infantil, factores de riesgo, prematuridad, primera infancia, extensión, empoderamiento familiar

Abstract: Since 2016, the Department of Conceptualization of Special Education at Universidad Estatal a Distancia has been developing the outreach project "UNED in action: Promoting new opportunities for children exposed to biological or social risk factors." The objective is to promote educational actions to train and empower families and caregivers of preterm children in early care (Atención Temprana, AT) with biological and social risk factors between ages 0 - 6 for their comprehensive development. Families of preterm children get orientation, especially considering that $10 \%$ of births in Costa Rica are preterm. Theory indicates that AT can revert the effects of this risk factor. Families need to understand what AT is, but they cannot always have access to the recommended practices or are unaware of their importance, fact that is accentuated outside the Greater Metropolitan Area where related services are limited or inexistent. University outreach programs allow for joint guidance and instruction with the families, which empowers them as change agents that overcome the traditional paradigm regarding early childhood stimulation conventionally framed under a clinical model where the specialist was the only one bestowed with knowledge. Via a methodological line of action research, the project offered workshops based on games and family learning. From 2017 to 2019, it was worked with eighty-eight participants in different provinces. The success element that favored family empowerment was basing the project on AT, promoting daily activities as learning experiences, the very philosophy of the research that originated the process.

Keywords: children development, risk factors, preterm birth, early childhood, outreach, family empowerment.

Resumo: A Cátedra de Conceptualização da Educação Especial da Universidad Estatal a Distancia vem desenvolvendo o projeto de extensão "UNED em ação: Promovendo novas oportunidades para crianças expostas a fatores de risco biológico ou social" desde 2016. O objetivo é promover ações educacionais para capacitar e empoderar famílias ou cuidadores de crianças com fatores de risco biológico e social entre 0 e 6 anos em atendimento precoce (ECC) (AT atención temprana) para seu desenvolvimento integral. O objetivo é orientar a famílias de bebês prematuros, considerando que em Costa Rica 10\% dos nascimentos são prematuros (Gonzáles, 2018). A teoria indica que a AT pode reverter os efeitos deste fator de risco. As famílias precisam entender o que é a AT, mas nem sempre são capazes de aceder as práticas recomendadas ou desconhecem sua importância, principalmente fora da Grande Área Metropolitana onde estes serviços são limitados ou inexistentes. A extensão universitária permite a capacitação conjunta com as famílias, empoderando-as como agentes de mudança do paradigma tradicional de estimulação enquadrado em um modelo clínico onde o especialista tinha o conhecimento, para um de AT que quebra os paradigmas tradicionais. O projeto assume uma linha metodológica de pesquisa-ação mediante uma oficina baseada em jogos e brincadeiras, e no aprendizado familiar. De 2017 a 2019 trabalhamos com 88 participantes em diferentes províncias. O elemento de sucesso que favoreceu o empoderamento familiar foi enquadrar o projeto a partir da AT, promovendo atividades diárias como experiências de aprendizagem, filosofia da investigação que originou o processo.

Palavras-chave: Desenvolvimento infantil, fatores de risco, prematuridade, primeira infância, extensão, empoderamento familiar. 


\section{INTRODUCCIÓN}

Un nacimiento es un acontecimiento de gran importancia para la vida familiar e implica nuevas tareas y retos. Ninguna familia está realmente preparada para este evento, más aún cuando se presentan factores de riesgo y en particular cuando ocurre en forma prematura. La dinámica cambia debido a los cuidados especializados que requiere el bebé y para los que no estaba organizada, todo se centra en la sobrevivencia del infante.

Barra y Alvarado (2012) consideran que un niño presenta alto riesgo o vulnerabilidad neurobiológica cuando existe una mayor probabilidad de mostrar alteraciones en el desarrollo ocasionadas por antecedentes pre, peri o postnatales. Entre estos factores de riesgo biológico y social cabe mencionar el bajo peso al nacer por prematuridad, la hipoxia en el período perinatal, la oxigenoterapia intensa y prolongada en prematuros, el consumo de drogas o la desnutrición de la madre durante el embarazo.

Según datos de la Organización Mundial de la Salud, cada año nacen 15 millones de niños prematuros en el mundo (Organización Mundial de la Salud, 2018). En Costa Rica, de los 78000 nacimientos que ocurren anualmente, de acuerdo con datos de la Caja Costarricense de Seguro Social, 10\% corresponde a la niñez prematura (González, 2018).

Diferentes autores plantean que el concepto de prematuridad se refiere al bebé que nace antes de las 37 semanas de gestación. El nacimiento anticipado lo vuelve vulnerable al entorno por el bajo peso y tamaño, así como por la inmadurez de su sistema en general. En este contexto, el concepto de edad corregida es importante de considerar, puesto que "sería la edad que tiene el niño si hubiera nacido el día que cumplía la semana 40 semana de EG [edad gestacional]" (Caserío \& Pallás, 2009, p. 445). Este término posibilita la valoración del desarrollo neurológico y físico partiendo de la edad que realmente tiene el infante al considerar las semanas de gestación cumplidas al momento de nacer.

Los avances científicos y tecnológicos, así como las condiciones hospitalarias, favorecen la supervivencia de bebés cada vez más prematuros y que puedan ir evolucionando en forma satisfactoria. Aunado a ello, "el involucramiento activo de todos los miembros del núcleo familiar, durante el período de recuperación de la madre y el neonato es vital" (González, 2018, párr. 9).

El panorama de mayores posibilidades de supervivencia es alentador, sin embargo, contrasta con el hecho de que la prematuridad es el principal factor de riesgo biológico perinatal. Tal y como lo establecen Quirós, Alfaro, Bolívar \& Solano (2016) "la prematuridad constituye una de las principales causas de morbimortalidad neonatal y es responsable de un gran porcentaje de secuelas infantiles" (p. 76). Esta exposición a factores de riesgo biológico en las etapas pre, peri o postnatal puede derivar en una condición de discapacidad. "Muchos de los bebés prematuros que sobreviven sufren algún tipo de discapacidad de por vida, en particular, discapacidades relacionadas con el aprendizaje y problemas visuales y auditivos" (Organización Mundial de la Salud, 2018, párr. 7).

En el momento del nacimiento se dispone, aproximadamente, de cien millones de neuronas, pero hay pocas conexiones entre sí. La etapa de los 0 a los 3 años constituye un período crítico del desarrollo, en el que el cerebro presenta mayor plasticidad. Es una ventana de oportunidad que posibilita crear nuevas conexiones neuronales mediante el proceso de sinapsis, hasta llegar a establecer una red. Las conexiones pueden variar e incrementarse en respuesta a estímulos dados. Es aquí donde el ambiente juega un papel importante. Si el infante recibe la atención temprana y oportuna, va a incidir positivamente en su crecimiento y desarrollo. Se van a generar más conexiones cerebrales que favorecen la adquisición de aprendizajes, pero también, permite revertir los posibles efectos de los factores de riesgo a los que el bebé se vio expuesto por la prematuridad; por cuanto el cerebro crea otras rutas de aprendizaje alrededor de la zona donde ocurrió alguna lesión. Si no recibe esta atención o no es apropiada, las neuronas mueren y no son sustituidas por otras (Flores, 2014). 
La atención temprana, entonces, "se convierte en una estrategia de prevención y promoción [...]que debe ser iniciada desde el momento que el niño nace, considerando acciones educativas hacia la sociedad, o la persona con quien interactúa y también quien estimula al niño" (Ayala \& Laos, 2016, p. 11).

La familia asume un papel protagónico desde la atención temprana que favorece el desarrollo integral del infante nacido prematuramente. Los enfoques recientes en esta área se orientan a que los cuidadores "se conviertan en corresponsables de las decisiones y verdaderos agentes de cambio en el entorno natural del niño" (García-Sánchez, 2014, p. 290).

Es en el contexto de la cotidianeidad donde el niño aprende, a lo largo del día y basado en la repetición de las actividades. Los familiares y cuidadores pueden proporcionar oportunidades de aprendizaje que sean contextualizadas e integrales. Señala García-Sánchez (2014), "el niño puede experimentar y esforzarse en una capacidad motora, por ejemplo, a la vez que se está buscando utilizar su lenguaje, comunicarse, y se está desarrollando habilidades sociales. Lo cual facilita el aprendizaje natural del niño [...]" (p. 295).

De esta manera, se destaca el reconocimiento del papel de la familia y los cuidadores en la atención del desarrollo integral de los infantes en la etapa de 0 a 3 años y, en particular, de los nacidos en forma anticipada, "Chávez (2014), Escalona \& López (2011), Moreno (2011) y Garrido (2015) enfatizan en la necesidad de que estén informados y se capaciten en este tema, lo cual conlleva al empoderamiento familiar" (Garrido, Madriz \& Antón, 2018, p. 493).

En el marco referencial la Cátedra de Conceptualización de la Educación Especial de la Universidad Estatal a Distancia (UNED) asume el compromiso con las familias de la niñez expuesta a factores de riesgo, en particular, con la nacida de forma prematura.

Desde el año 2011, se desarrollan investigaciones en este campo que llevan al lanzamiento en el año 2019 de una herramienta tecnológica para familias y cuidadores. Está orientada, como su nombre lo indica, a la detección, estimulación y atención temprana (DEAT) para la prevención de la discapacidad en infantes costarricenses con una condición de riesgo biológico (Loría, 2019). La herramienta tecnológica consta de una serie de escalas del desarrollo infantil con sus respectivos tutoriales audiovisuales entre las edades de un mes hasta los 48 meses. Comprende, además, infografías de signos de alerta para que las familias puedan monitorear el desarrollo de sus hijos y acompañarlos en el proceso, así como empoderarlas en atención temprana para facilitar la derivación oportuna de ser necesario.

De la línea investigativa de la DEAT, se desprende que se debe dar respuesta a las necesidades de las familias y es por medio de la extensión universitaria que se pueden atender las mismas. Por tanto, se desarrolla desde el año 2016 el proyecto “UNED en acción: Promoviendo nuevas oportunidades para la infancia expuesta a factores de riesgo biológico o social".

En este sentido, la extensión es una de las funciones sustantivas de la UNED que desde su modelo pedagógico la define como: "las acciones orientadas a promover la mejora de la calidad de vida de personas y comunidades en general a través del desarrollo de programas y proyectos" (CIE, 2011, párr. 7).

Las familias costarricenses, principalmente las que viven fuera de la Gran Área Metropolitana carecen de servicios de atención temprana para la infancia prematura, lo cual las coloca en una posición de vulnerabilidad; por tanto, resalta la importancia de la extensión universitaria como estrategia para cerrar la brecha y crear oportunidades por medio del empoderamiento que deriva del conocimiento.

Por ende, el objetivo de este proyecto de extensión es promover acciones educativas para capacitar en atención temprana a las familias y personas encargadas del cuido de infantes de 0 a 6 años con factores de riesgo biológico y social, con el fin de favorecer su desarrollo integral. Esto bajo una filosofía de acompañamiento de aprendizaje mutuo que valida la experiencia y el conocimiento de las redes familiares en procesos de atención temprana desde la cotidianeidad y de forma lúdica en diferentes zonas del país. 
Lo anterior, por el grado de involucramiento de las familias y su empoderamiento para que asuman las prácticas recomendadas en atención temprana como equipo, por medio de un taller de formación. Se rompe así con el esquema tradicional de la estimulación temprana dado que la metodología es participativa, con procesos de construcción y deconstrucción para la validación de las experiencias de las redes familiares.

Los proyectos de extensión no siempre trascienden al trabajo con el colectivo denominado familia; en los que cada uno de sus miembros es igual de relevante y donde se desmitifique tanto que el cuido de los infantes es responsabilidad de la mujer como que la atención temprana es una tarea externa al quehacer de la cotidianeidad. Esto permitiría romper varios paradigmas a la vez, lo que da origen incluso a un cambio de nombre del taller de formación: "Jugamos, aprendemos y nos empoderamos en la atención temprana".

\section{MATERIALES Y MÉTODOS}

El proyecto de extensión se enmarca en el paradigma naturalista puesto que su objetivo es la inmersión en los contextos que permita que los participantes sean sujetos activos. El enfoque es cualitativo bajo un diseño de investigación-acción. Desde este diseño los protagonistas, con sus acciones, transforman la realidad social. Es así como, desde el proyecto, se pretende que los participantes puedan generar, mediante su empoderamiento como agentes de atención temprana, las acciones que contribuyan al mejoramiento de las condiciones de la niñez nacida de forma prematura.

Al partir de la "[...] construcción, reconstrucción y de-construcción del conocimiento [...]" (Agrelo, 2011, párr. 2), se adopta una metodología participativa. La elección radica en que sus características responden a los objetivos del proyecto: lúdica, interactiva, creativa y flexible, fomenta la conciencia grupal, práctica, formativa, comprometida y comprometedora (Agrelo, 2011).

El componente lúdico es uno de los principales elementos del taller por cuanto el juego es el motor del aprendizaje tanto en la infancia como en la adultez. Se pretende generar un espacio interactivo y formativo en el que los participantes, por medio del juego, puedan confrontar sus ideas acerca de la importancia de la atención temprana y las prácticas que en esta área han venido desarrollando con sus hijos. A partir de ahí, la reflexión individual y colectiva ha de conducir a una nueva mirada que los empodere como agentes de atención temprana de los infantes.

La metodología favorece la cohesión grupal de las familias; el sentido de pertenencia y el apoyo que puedan darse entre sí al compartir vivencias similares como el nacimiento en forma prematura de sus hijos. Lo anterior contribuye además a asumir un compromiso con el proceso y la puesta en práctica en el hogar.

Asimismo, el taller es una de las técnicas más usadas desde la investigación-acción. Es un "lugar de coaprendizaje, donde todos sus participantes construyen socialmente conocimientos y valores, desarrollan habilidades y actitudes, a partir de sus propias experiencias" (Secretaría de Educación de Gobierno del Estado, 2011, p. 12).

Para el proceso de planificación del taller, se consideró que, de acuerdo con Garrido (2015), "un proceso de sensibilización y capacitación en atención temprana debe combinar los elementos teóricos con experiencias vivenciales y prácticas" (p. 99); aunado a ello, se debe contemplar la detección de las necesidades y las expectativas de los participantes en el área de la atención temprana, así como las experiencias o conocimientos previos.

El trabajo del taller en primera instancia se orientó hacia el desarrollo de acciones que favorecieran en los asistentes su empoderamiento personal y grupal, y en propiciar cambios en las actitudes y percepciones; 
finalmente, a que las familias participantes sean conscientes de la importancia de la atención temprana para la niñez prematura.

El taller consta de una serie de sesiones en las que se utilizó la técnica de la observación participativa, que ha permitido a las extensionistas realizar y participar de las actividades a la vez que se registra información relevante. Se realizaron grupos focales a partir de una guía de cuatro preguntas generadoras. Durante las sesiones se tomaron fotografías y se grabaron algunos videos para registrar la información y como evidencia.

Al término de cada sesión, se aplicó a los adultos participantes una evaluación para determinar el efecto de los talleres en sus prácticas de atención temprana, posibles cambios que pueden incorporar en ellas y las necesidades de capacitación en el área. El instrumento utilizado consta de un cuestionario de dieciséis ítems con escala de Likert y cuatro preguntas de respuesta abierta.

Además, la información de cada sesión fue registrada en una bitácora del diario de campo, las cuales se transcribieron para la sistematización de los talleres y de todos los instrumentos aplicados.

El contenido formativo de los talleres se dirigió a temas del interés de las familias: neuroplasticidad, áreas del desarrollo y actividades para favorecer las diferentes destrezas, lenguaje, estimulación sensorial, manejo de límites y emociones. Todos enmarcados en el paradigma de la atención temprana. También, se entregó en cada sesión una carpeta con las actividades desarrolladas, ejercicios adicionales para realizar en el hogar y documentación complementaria sobre el tema.

Siguiendo la propuesta de Garrido (2015), cada sesión de dos horas de duración se estructuró en cuatro momentos: motivación, exposición, aplicación y evaluación:

- En el primero se desarrollaron actividades de animación o de integración grupal, se compartieron expectativas y experiencias previas.

- En la parte de exposición, se desarrolló en forma breve el tema central de la sesión.

- La aplicación fue el de mayor duración al ser el espacio en el que las familias realizaron las actividades propuestas con los infantes bajo la guía y acompañamiento de las extensionistas, las que también podían modelar la forma de ejecución y la interacción con el niño.

- Finalmente, en la evaluación, por medio de un instrumento escrito, se buscó comprobar los conocimientos adquiridos y la opinión de los participantes del trabajo realizado en su práctica cotidiana en el hogar.

El componente participativo y lúdico se mantuvo presente a lo largo de la sesión, tanto con los adultos como con los infantes, y se propició que se involucrara en los cuatro momentos.

La población meta del proyecto de extensión está conformada por familias y personas encargadas del cuido de infantes expuestos a factores de riesgo con edades entre 0 y 6 años. En el período 2017-2019 se focalizó en la niñez prematura con edades entre 6 y 36 meses y sus familias, además de funcionarias de centros de cuido infantil en diferentes zonas del país.

Para captar a las familias participantes se emplearon estrategias tales como: identificación de familias participantes en la DEAT, divulgación en redes sociales, recursos comunales para el contacto y derivación, por ejemplo, el personal médico de hospitales, así como con docentes del servicio de Estimulación Temprana del Centro de Educación Especial de San Ramón. Se han desarrollado coordinaciones con la Fundación para padres de niños prematuros Costa Rica (FUNDAPREMA), con quienes además se participó en charlas durante las actividades para la conmemoración del Día Mundial del Prematuro; a partir de estas actividades también se logró captar a los asistentes. 
Para una mayor participación de redes familiares, los talleres se realizan en un horario ajustado a las necesidades de las familias fuera de horarios laborales.

En relación con la garantía del tratamiento ético de la información, se utilizó un formulario de consentimiento informado. A todos los participantes se les explicó el proyecto, sus objetivos y alcances. Por involucrar infantes, los progenitores autorizaron el manejo de imágenes de sí mismos y de sus hijos.

\section{RESULTADOS Y DISCUSIÓN}

Entre los años 2017 a 2019 se han impartido dieciséis sesiones del taller en las zonas de San José, Puntarenas, Palmares y San Ramón con la participación de 62 familias de infantes con edades entre los seis y los treinta y seis meses. Además, se desarrollaron dos sesiones con veintiséis funcionarias de un Centro de Cuido y Desarrollo Infantil (CECUDI) y de una guardería pública en Cartago que en conjunto atienden una población infantil de ciento cinco infantes.

En el año 2020, por la pandemia del COVID-19, las sesiones del taller presencial se suspendieron en acatamiento de las órdenes sanitarias del país y se incursionó en la virtualidad para impartirlas.

Un aspecto importante del éxito en la participación del taller es el empleo de una metodología que favorezca que los participantes puedan sentirse capaces de desarrollar la atención temprana y fomentar la motivación. Se procura ayudarles, desde la planificación, a que puedan identificar cómo mejora el vínculo de toda la red familiar y facilita la adquisición de habilidades en la población de la primera infancia, lo cual se vuelve una forma de realimentación de la labor realizada. Enfatiza en que las familias deben ser partícipes activas de su propio aprendizaje que derive en el desarrollo de competencias para buenas prácticas en el hogar (García-Sánchez, 2014).

Para la discusión de los resultados obtenidos se abordaron dos líneas relacionadas con los orientados hacia las familias y los que se centran en la niñez.

En relación con las familias, partiendo del objetivo del proyecto se desprenden tres elementos para el análisis de los resultados: las acciones educativas, la sensibilización y la capacitación.

La valoración de los resultados de los procesos de sensibilización desarrollados a lo largo de las sesiones del taller mostró que $100 \%$ de los participantes coincidió en que les ayudaron a comprender la importancia de la atención temprana, así como a entender el concepto y la necesidad de potenciar las diferentes áreas del desarrollo en sus hijos de una manera más divertida y permanente. Lo anterior, al conocer que, desde la plasticidad cerebral y la ventana de oportunidad desplegada en los primeros años de vida, es que el cerebro puede ser modificado química, anatómica y fisiológicamente en un ambiente enriquecido por diversas experiencias (Muñoz \& Almonacid, 2017). De igual manera, permite la adquisición de aprendizajes y habilidades específicas, así como una recuperación funcional al limitar la extensión y consecuencias de lesiones pre, peri o postnatales.

Como ejemplo de lo anterior, una de las participantes de Cartago, señaló lo siguiente: "El conocimiento que un niño adquiere depende mucho de la atención brindada" mientras una madre, en uno de los grupos focales de San José expresó: "Conocer lo importante que es jugar con mi bebé con objetos sencillos para que aprenda a desenvolverse y realizarse, darle confianza en sí misma desde una etapa temprana".

Referente a favorecer cambios en actitudes, percepciones y las prácticas de atención temprana realizadas, los padres de familia expresaron en los instrumentos de evaluación aplicados "ser más dinámico, tener más paciencia" y "dedicar más tiempo". Una de las participantes, madre de trillizas, mencionó lo siguiente: 
"Dejar de lado la preocupación diaria y ponerlas a explorar más distintas cosas"; otra señaló que debe "informarse más, preguntar y poner en práctica lo aprendido".

En las evaluaciones aplicadas, 100\% de los participantes señalaron sentirse capaces de ofrecer al infante atención temprana en las diferentes áreas del desarrollo. Otros aspectos a los que hicieron mención como cambios por realizar son: "Entender que los niños tienen una evolución diferente en tiempo, a nivel social, entre otros"; "Hablar más con él de manera que su lenguaje mejore y se frustre menos; crear espacios en la casa que faciliten las actividades familiares y no solo las monoparentales".

El hallazgo señalado se valoró como muy importante ya que apunta a uno de los ejes centrales de la propuesta y que la hace innovadora, es la incorporación de los miembros de la familia en las actividades desarrolladas. Se ha buscado romper el paradigma que enmarca los roles tradicionales de género que atribuyen la responsabilidad del cuido y crianza del infante a la madre. En este sentido, Campabadal \& Richmond (2009) exponen que en las "familias que se apegan a roles tradicionales de género [...] es probable que la madre deba asumir en solitario la responsabilidad del hijo o hija [...] y lo que ello implica" (pp. 44-45). Desde ese enfoque aplica la atención temprana para romper con el patriarcado y la idea de que el cuido de las personas menores de edad es una responsabilidad inherente al género femenino.

El proyecto pretende apartarse de estas premisas al enmarcarse desde la atención temprana que privilegia la participación de las redes familiares. De esta manera, durante las sesiones realizadas, se contó con la presencia y participación de miembros de la familia extensa, además de ambos progenitores. Como lo expresa un padre de Puntarenas, en uno de los grupos focales: "Motivar a otros miembros de la familia a participar en las actividades que favorezcan el desarrollo del niño".

"Aparte de los ratos de juego también aprovechar otras actividades cotidianas", esta expresión de otro progenitor refleja la filosofía del taller que es convertir todas las actividades de la vida diaria en una experiencia de aprendizaje. De igual manera, una madre indicó: "Exponerlo a situaciones de la vida cotidiana y explicarle qué es; interactuar con el bebé para hacerlo partícipe de las actividades".

Aunado a lo anterior, se generó un cambio en la creencia de los participantes en cuanto a la necesidad de sacar un espacio y un tiempo determinado para la ejecución de las actividades que favorezcan el desarrollo infantil, al comprender que han de estar inmersas en la vida familiar cotidiana. Como evidencia, 100\% de los asistentes afirmaron que pueden poner en práctica en la casa las actividades realizadas en el taller.

En este sentido, Madriz, Antón, Palomares, Soto y González (2016) plantean lo siguiente:

Si consideramos que el desarrollo se produce a través de la interacción y que los aprendizajes adquieren significado en el proceso natural, el ambiente familiar es el espacio ideal para promover desde la cotidianeidad, experiencias ricas y variadas que le permitan al niño alcanzar al máximo el potencial que poseen. (p. 5)

El ámbito familiar es por excelencia el espacio en el que el infante se desenvuelve, donde se siente motivado y puede aprender rodeado de las personas significativas. Por esta razón, en la atención temprana la familia es la protagonista. El hogar brinda oportunidades de aprendizaje continuas, variadas y permanentes a lo largo del día en la interacción con los diferentes miembros de ésta, en contraposición con el espacio y el tiempo limitados que pueda recibir en las sesiones de "estimulación temprana" en un centro educativo o de salud.

Un aspecto en el que se enfatizó en las sesiones fue que los participantes pudieran incorporar la constancia en sus prácticas de atención temprana en el hogar. "Las oportunidades de aprendizaje contextualmente mediado se repetirán a lo largo del día y de la semana, una y otra vez, y en situaciones diferentes, favoreciendo el aprendizaje y su generalización" (García-Sánchez, 2014, p. 292). 
Otra creencia que se logró modificar con el proceso de sensibilización fue considerar que para la atención temprana se requiere de juguetes caros o especializados y que se realiza en forma externa al hogar. Es así como varios participantes coincidieron al mencionar "lo práctico de utilizar cualquier material que hay en el hogar", "con materiales accesibles y que se tienen en casa se puede realizar una bonita y entretenida actividad" y "utilizar materiales más sencillos para la facilidad de aprendizaje de los niños".

El anterior hallazgo es otra fortaleza del proyecto ya que los miembros de la familia se motivaron y se sintieron en capacidad de realizar estrategias de atención temprana pues reflexionaron sobre la posibilidad de emplear recursos y materiales de uso cotidiano en el hogar. El descubrimiento de reconocerse en capacidad de desarrollar actividades y tener los medios para hacerlo de forma expedita fue expuesto en las evaluaciones por la totalidad de los participantes. De esta manera, se han empoderado como agentes de atención temprana.

Esto se reflejó, además, cuando se les consultó a los participantes por las actividades que pudieron poner en práctica de una sesión a otra del taller. En su mayoría, mencionaron que lograron realizar en el hogar las que se hicieron en la sesión e incluso las adaptaron a las necesidades específicas de sus hijos o a las condiciones familiares. En este sentido, y reforzando lo expuesto anteriormente, se evidenció que las actividades que con más facilidad replicaron fueron aquellas en las que se utilizaban materiales de uso cotidiano o que del todo no los requerían, algunos señalamientos fueron :"Le pusimos arena en una caja e hicimos masa sensorial", "Cuando está inquieto le canto", esto último en alusión a que, en la sesión ante el llanto del niño una de las extensionistas le empezó a cantar y, para el asombro de sus padres, se calmó.

Además, la metodología desarrollada generó entre las familias participantes, interacciones socioafectivas, actitudes de motivación, empatía y de apoyo. Los asistentes expresaron sentirse en la confianza de compartir sus experiencias, preocupaciones y dudas en torno al desarrollo y crianza de sus hijos; entre ellos se daban recomendaciones, se alentaban e incluso reconocían, de una sesión a otra, los avances de los demás infantes. Se identificaron elementos de empoderamiento grupal.

Siguiendo a Galván (2017), en el empoderamiento las personas o grupos refuerzan su confianza y capacidades, lo que les permite asumir un papel protagónico y de compromiso en los procesos de cambio en las situaciones del diario vivir. Favorece el accionar autónomo, la toma de decisiones y la responsabilidad. La familia empoderada se siente en la capacidad de poder brindar los apoyos que el infante requiere para su desarrollo integral.

Para alcanzar el empoderamiento, es importante también que las familias tengan acceso al conocimiento, por esta razón, en las sesiones se desarrolló un espacio de capacitación. En este sentido, los participantes expresaron haber adquirido conocimientos que les permitieron, por ejemplo, crear espacios para el desarrollo del lenguaje de sus hijos, establecer límites y manejar los berrinches. De igual manera, esta necesidad de aprender se vio reflejada en las expectativas de los padres al asistir a las sesiones del taller, puesto que expresaron: "Siento que es muy importante aprender más cuando son prematuros" y "Me interesa saber cómo aplicarlo a mi hija en la casa".

En esta área, un hallazgo obtenido fue que las familias tenían diferentes grados de conocimiento sobre temas relacionados con la atención temprana y la prematuridad. Uno de esos aspectos fue el concepto de edad corregida. Se encontró que las familias de Puntarenas y San Ramón tenían más conocimiento que las de San José debido a que recibieron orientación médica al respecto.

En relación con el material escrito facilitado en cada sesión, $100 \%$ de las familias expresaron que era claro y práctico. Señalaron que les permitía repasar los conceptos y actividades que se realizaron, aclarar dudas $y$, algo fundamental, poder compartirlo con otros miembros e incluso con otras familias. Este resultado es primordial para los objetivos del proyecto ya que extiende el alcance del taller más allá de los participantes. 
La segunda línea de análisis y discusión de los resultados está centrada en la niñez que asiste a las sesiones. En este sentido, se evidenció que algunos de los infantes adquirieron nuevas habilidades y destrezas de las diferentes áreas del desarrollo durante la ejecución de las actividades, así como luego de las sesiones, lo cual fue expresado por los miembros de la familia, quienes se mostraron sorprendidos de los logros.

Las familias manifestaron su satisfacción y gratitud por el aprendizaje obtenido, el espacio compartido, así como por el apoyo y el respaldo recibidos. Además, en los instrumentos de evaluación aplicados, $100 \%$ de los asistentes señalaron sentirse satisfechos con aspectos de organización tales como logística, espacio utilizado, iluminación, duración y horario. También, expresaron sentirse preparados y comprometidos con sus hijos para la atención temprana en el hogar. Uno de los asistentes, en relación con el taller expresó: "Darlo más seguido y en mayores lugares a nivel país ya que son de suma importancia".

Es evidente el interés que tienen las familias por aprender y poder desarrollar competencias para la atención temprana de los infantes nacidos de forma prematura. Esta necesidad es aún más palpable en zonas alejadas de la capital, donde el acceso a capacitaciones y a servicios de esta índole es limitado.

La experiencia vivida con el grupo de Puntarenas reflejó la necesidad de capacitación, pero a la vez es un ejemplo del potencial de organización que existe en las comunidades. Al realizar la divulgación del taller en redes sociales, personas de zonas fuera de la Gran Área Metropolitana lamentaron no poder asistir por realizarse en San José. La conformación de del grupo surgió de la iniciativa de una madre de familia de la zona quien contactó a las extensionistas y fue agente activa para organizar a los participantes.

La extensión y la acción social cobran un papel preponderante al posibilitar que iniciativas como este proyecto puedan llegar a diferentes lugares del país en forma gratuita y solventar una necesidad vital de las familias costarricenses: potenciar el desarrollo integral de la niñez prematura a partir de estrategias de atención temprana desarrolladas en el seno familiar.

Es importante señalar que el objetivo del proyecto ha trascendido a otras actividades vinculantes. La experiencia alcanzada hasta el momento ha sido expuesta en dos congresos internacionales en Granada, España y en Cuernavaca, México y ambas ponencias han sido publicadas. En el ámbito nacional también se ha presentado una ponencia en el I Congreso Interuniversitario de Extensión y Acción Social. Además, se ha elaborado una serie de tres infografías bajo el título "La familia se empodera ante el nacimiento de un niño prematuro" para su difusión a nivel nacional y por medio de la DEAT.

\section{CONCLUSIONES}

La fortaleza que ha asegurado a este proyecto la permanencia y la continuidad en el tiempo es atribuible al hecho de que se enmarca en un contexto que trasciende la estimulación tradicional y se enmarca en la atención temprana que, aunque es un paradigma de reciente implementación en Costa Rica, el proyecto nace con esa visión porque está ligado a la DEAT cuya filosofía es convertir cada actividad de la cotidianeidad en una experiencia de aprendizaje.

Si bien la nomenclatura de la UNED agrupa a este tipo de actividades en lo que se conoce como extensión, es realmente una experiencia de acción social que empodera a la Cátedra de Conceptualización de la Educación Especial en la responsabilidad social que tienen las universidades de aportar a las poblaciones vulnerables el insumo necesario para la realimentación que mejore su calidad de vida.

Desde la extensión ha sido posible el acercamiento a familias con infantes nacidos de forma prematura en diferentes zonas del país. La labor desarrollada por el proyecto favoreció el empoderamiento familiar como estrategia de atención temprana de poblaciones vulnerables, así como con el romper con algunos paradigmas tradicionales de estimulación. 
Por otra parte, las familias participantes reconocen y asumen el compromiso por desarrollar las actividades dentro del enfoque de atención temprana, más allá del esquema tradicional de la estimulación, superando la creencia de que ésta es una labor que compete exclusivamente a la madre.

Aunado a lo anterior, el proyecto ha permitido visualizar el riesgo biológico y social como una condición de vulnerabilidad, en este caso específico, la prematuridad para la niñez costarricense, por ende, es un nicho para que las universidades puedan realizar proyectos de extensión que empoderen y mejoren la calidad de vida de las familias.

Es decir, los procesos de sensibilización, capacitación y ejecución práctica han venido a modificar la forma en que se venía realizando la atención de los niños en el hogar en cuanto al tipo de actividades, frecuencia, repetición y continuidad. Los participantes fortalecen sus competencias para el desarrollo de buenas prácticas de atención temprana en la cotidianeidad familiar.

Además, la incorporación del elemento lúdico en la metodología ha permitido que, con las actividades de atención temprana, la familia se reencuentre y fortalezca sus vínculos.

Por otra parte, la experiencia de Puntarenas, desde la extensión y la acción social, constituye una muestra de la forma en que las fuerzas comunales, en este caso, grupos de familias, se pueden organizar y gestionar la consecución de los medios para satisfacer una necesidad sentida por ese colectivo, en este caso, para el desarrollo de acciones de atención temprana para la niñez prematura desde el hogar.

Otro aspecto que también trascendió los alcances del proyecto es el hecho de que algunas de las familias asistentes, no sólo se convirtieron en agentes de atención temprana en sus hogares, sino que fueron más allá al compartir con otros grupos familiares la experiencia y orientarlos hacia las prácticas recomendadas.

Los proyectos siempre tienen un objetivo, pero un indicador de éxito es cuando no sólo se alcanza el objetivo meta, sino que se trascienden los límites, por ejemplo, con las publicaciones y ponencias a nivel internacional y nacional, así como la elaboración de la serie de infografías para la divulgación en el país.

Como un elemento que enriquecería el proyecto, se podría explorar, a la hora de vincularse con los grupos familiares, las prácticas recomendadas de atención temprana que vienen desarrollando, con el fin de determinar si en Costa Rica se ven influenciadas por elementos culturales, étnicos o sociales.

Si bien, hasta el momento, se evidencia el logro del objetivo propuesto en el proyecto e incluso ha trascendido, el reto desde la extensión universitaria y la acción social es poder llegar a familias de comunidades distantes y de zonas que presentan además otros factores de vulnerabilidad. La dupleta atención temprana y extensión social debe continuar recorriendo el camino iniciado en el año 2016 como forma de saldar una deuda con las familias de la niñez expuesta a factores de riesgo.

\section{REFERENCIAS}

Agrelo, A. (2011). Metodología Participativa. [Diapositiva de prezi]. Recuperado de https://www.slideshare. net/andreagrelo/metodologa-participativa

Ayala, J.A. Laos, A.J. (2016). Conocimiento y prácticas sobre estimulación temprana en madres de niños menores de 1 año, Hospital Barranca Cajatambo 2016 (tesis de licenciatura en línea, Universidad Nacional Santiago Antúnez de Mayolo) Recuperado de http://repositorio.unasam.edu.pe/bitstream/ handle/UNASAM/1846/T033_46637283_T.pdf?sequence=1\&isAllowed=y

Barra, L., Alvarado, R. (2012). Frecuencia de riesgo neurobiológico en recién nacidos. Revista Chilena de Pediatría, 83(6), 552-562. Recuperado de https://scielo.conicyt.cl/scielo.php?script=sci_arttext\&p id $=$ S0370-41062012000600004 
Campabadal, M., Richmond, V. (2009). Redes de apoyo para la autodeterminación de las personas con discapacidad. Editorial EUNED: San José, Costa Rica.

Caserío, S. \& Pallás, C.R. (2009). Seguimiento del prematuro/gran prematuro en atención primaria. Pediatría de Atención Primaria, 6 (17), 443-450. Recuperado de https://www.researchgate.net/ publication/40496861_Seguimiento_del_prematurogran_prematuro_en_Atencion_Primaria

Centro de Investigación y Evaluación Institucional. (2011). Unidad 2: Modelo pedagógico y áreas sustantivas de la UNED. (Multimedia). Recuperado de https://multimedia.uned.ac.cr/pem/pedagogia_universitaria/paginas_unidad2/modelo_pedagogico.html

Flores, J.M. (2014). Efectividad del programa de estimulación temprana en el desarrollo psicomotor en niños de 0 a 3 años. Vida's Centro de la Familia. (tesis doctoral, Universidad de Trujillo). Recuperado de http:// dspace.unitru.edu.pe/bitstream/handle/UNITRU/5613/Tesis\%20Doctorado\%20-Jacqueline\%20 Flores\%20Aguilar.pdf?sequence=1\&isAllowed=y

Galván, F. (2017). Empoderamiento: una estrategia de liderazgo. Recuperado de https://www.ebc.mx/ educacioncorporativa/articulos/empoderamiento-una-estrategia-de-liderazgo.php\#: :text=El\%20 empoderamiento\%20organizacional\%20se\%20refiere,las\%20operaciones\%20de\%20la\%20 organizaci\%C3\%B3n\%2C

García-Sánchez, F.A. (2014). Atención temprana: enfoque centrado en la familia. En Asociación Española de Logopedia, Foniatría y Audiología (Eds.), Logopedia: evolución, transformación y futuro (pp. 286-302). Murcia, España: AELFA. ISBN 978-84-697-0765-4

Garrido, A.L. (2015). Análisis del alcance de un proceso de sensibilización y capacitación en atención temprana dirigida a cuidadoras de niños entre 0 y 12 meses que presentaron alguna condición de riesgo biológico neonatal en el Hogar de Vida para la Niñez para la promoción del desarrollo personal (tesis de maestría). Recuperado de http://repositorio.uned.ac.cr/reuned/bitstream/120809/1631/1/ Análisis\%20del\%20alcance\%20de\%20un\%20proceso\%20de\%20sensibilización\%20y\%20capacitación\%20en\%20atención\%20temprana\%20dirigido\%20a\%20cuidadoras\%20de\%20niños.pdf

Garrido, A.L., Madriz, L.M., y Antón, P. (2018). Buenas prácticas de sensibilización y capacitación en atención temprana como estrategia de empoderamiento familiar. En M.J. León \& T. Sola (Eds.), Liderando investigación y prácticas inclusivas (pp. 491-498). Granada, España: Editorial Universidad de Granada. ISBN 9788433862310

González, A. (17 de mayo de 2018). Casi 8 mil niños prematuros nacen cada año en Costa Rica. Amprensa.com. Recuperado de https://amprensa.com//2018/05/casi-8-mil-ninos-prematu ros-nacen-cada-ano-en-costa-rica/

Loría, M.F. (15 de junio de 2019). UNED presenta herramienta digital para atención y estimulación temprana en la niñez. El Sol de Occidente.com. Recuperado de https://elsoldeoccidente.com/enlinea/2019/06/ uned-presenta-herramienta-digital-para-atencion-y-estimulacion-temprana-en-la-ninez/

Madriz, L.M., Antón, P., Palomares, A., Soto, L., y González, V. (2016). Familias empoderadas-estimulación y atención temprana. [Diapositiva de prezi]. Recuperado de https://prezi.com/ztjm80be8t2s/ familias-empoderadas-estimulacion-y-atencion-temprana/

Muñoz, M.F., y Almonacid, A. (2017). Cognición, juego y aprendizaje: una propuesta para el aula de la primera infancia. Infancia, Educación y Aprendizaje, 1(1), 162-177. Recuperado de https://revistas. uv.cl/index.php/IEYA/article/view/576/592

Organización Mundial de la Salud. (2018). Nacimientos Prematuros. Recuperado de https://www.who. int/es/news-room/fact-sheets/detail/preterm-birth\#: :text=Se\%20considera\%20prematuro\%20 un\%20bebé,prematuros\%20(28\%20a\%2032\%20semanas)

Quirós, G., Alfaro, R., Bolívar, M., y Solano, N. (2016). Amenaza de parto pretérmino. Revista Clínica de la Escuela de Medicina UCR-HSJD, 1(1), 75-80. Recuperado de https://revistas.ucr.ac.cr/index.php/ clinica/article/view/23061/23287 
Secretaría de Educación de Gobierno del Estado San Luis Potosí. (2011). Guía para desarrollar talleres de orientación con padres y madres de familia. Recuperado de http://www.seslp.gob.mx//pdf/ taller2011-2012/tres/GUIA\%20DE\%20TALLERES\%20\%2018\%20DE\%20JULIO.pdf

Toasa, J. E. (2015). La importancia de la estimulación temprana en el desarrollo psicomotriz de los niños y niñas de 0 a 5 años que acuden a la consulta pediátrica en el Hospital General Puyo" (tesis de licenciatura en línea). Recuperado de http://repositorio.uta.edu.ec/handle/123456789/12205 